\title{
Chapter 15. Follow-up of CKD patients
}

(C) Japanese Society of Nephrology 2009

- It is important in the follow-up of CKD patients to slow worsening of the disease and to prevent CVD.

- In the case of $\mathrm{eGFR} \geq 50 \mathrm{~mL} / \mathrm{min} / 1.73 \mathrm{~m}^{2}$, primary care physicians manage $\mathrm{CKD}$, collaborating with nephrologists.

- In the case of eGFR $<50 \mathrm{~mL} / \mathrm{min} / 1.73 \mathrm{~m}^{2}$, primary care physicians and nephrologists manage CKD concurrently.

- A patient is recommended to be referred to nephrologists immediately after onset of abrupt increase of urinary protein or rapid decline of eGFR.

- Strategies of follow-up vary depending on primary diseases for CKD.

- Urinalysis, calculation of eGFR, and image testing are conducted at regular intervals to assess kidney function as well as to try to find CVD.

\section{Reasons for importance of CKD follow-up}

- Progression of each CKD stage toward end-stage kidney disease (ESKD) is accelerated as the stage advances. It is therefore necessary to confirm therapeutic effectiveness in order to slow CKD progression.

- Even in stages 1-3, the probability of death from cardiovascular disease (CVD) is greater than that of proceeding to ESKD.

- It is possible to slow the progression of CKD by lifestyle education and drug therapy, but regular followup is required to determine their efficacy.

- It has been evidenced that control of blood glucose as well as blood pressure and use of ACE inhibitors as well as ARBs is effective in suppressing CKD progression. Treatment of dyslipidemia or anemia or restriction of dietary protein also has similar effects.
Follow-up differences depend on primary diseases

- Diabetic CKD has a high prevalence of CVD and progresses rapidly in kidney function. Blood glucose should be controlled to keep HbA1c below 6.5\%. ECG and cardiac echography are recorded to prevent CVD development. At stage 5 of diabetes-related CKD, the kidney can degrade less insulin, resulting in better diabetes control. This apparent better control implicates worsened CKD.

- CKD due to hypertension, if at an early stage, can be improved through strict blood pressure control. ACE inhibitors or ARBs are particularly used as first-line agents.

- In case of CKD at stage 1-2 caused by chronic glomerulonephritis, if urinary protein excretion is $\geq 0.5 \mathrm{~g} /$ day, a patient is referred to nephrologists, who might carry out renal biopsy if feasible and determine a therapeutic approach based on histology of the biopsy specimen. Among CKD stage 3, cases with eGFR $<50 \mathrm{~mL} / \mathrm{min} /$ $1.73 \mathrm{~m}^{2}$ are referred to nephrologists for examination. Primary care physicians manage the case thereafter.

- Follow-up studies of CKD at stages 1-2 are delineated in Table 15-1.

- A urine specimen is examined for protein (as well as for microalbumin in diabetes) and is evaluated by urinary protein/urinary creatinine ratio. CKD progresses more rapidly as the amount of urinary protein increases.

- A CKD patient is examined for blood pressure at every visit, and also for $\mathrm{HbA} 1 \mathrm{c}$ if diabetic. Blood pressure is lowered below 130/80 $\mathrm{mmHg}$ in general or below 125/ $75 \mathrm{mmHg}$ in case of proteinuria $\geq 1 \mathrm{~g} /$ day. HbA1c is recommended to be less than $6.5 \%$ in diabetes. CKD progression is greatly affected by blood pressure and glycemic control. 
Table 15-1 Follow-up examinations at general physicians for stable patients with CKD stage 1 or 2

\begin{tabular}{ll}
\hline Variables & Frequency \\
\hline Blood pressure & Every visits \\
Proteinuria, urine creatinine & Every 3-6 months \\
Serum creatinine, eGFR & Every 3-6 months \\
Blood chemistry (total protein, albumin, electrolyte, lipids) & Every 3-6 months \\
HbA1C (when DM) & Every 1-3 months \\
X-p (chest, abdomen including lateral view) & Screening and annually \\
Ultrasonography, CT of the kidney & Screening and as needed \\
ECG & Screening and annually \\
\hline
\end{tabular}

- Blood analysis of concentrations of the following components varies among CKD stages: electrolytes including $\mathrm{Na}, \mathrm{K}, \mathrm{Cl}, \mathrm{Ca}$, and $\mathrm{P}$; urea nitrogen and uric acid; lipid including T-Chol, TG, LDL-C, and HDL-C; total protein and albumin. In CKD stages 4-5, electrolyte abnormalities such as hyperkalemia, hyperphosphatemia, and hypocalcemia emerge. It is noteworthy that hyperkalemia, in particular, may cause cardiac arrest due to ventricular arrhythmia.

- General blood panel is necessary. Erythropoietin production by the kidney is reduced as kidney function declines, leading to normocytic normochromic anemia. Furthermore, since bleeding tendency may emerge in stage 4-5 CKD, anemia due to blood loss from the gastrointestinal tract must be differentiated from iron-deficiency anemia ascribable to appetite loss. The presence of anemia requires the determination of serum iron, transferrin saturation (TSAT), and ferritin.
- At stage 3 or later, blood gas analysis is performed. $\mathrm{HCO}_{3}$ can be measured in a venous blood sample. $\mathrm{CKD}$, if complicated by metabolic acidosis, progresses faster and osteolysis is accelerated.

- Electrocardiogram is recorded to examine heart disease. If the test indicates suspected ischemic heart disease, further studies such as cardiac ultrasonography, cardiac muscle scintigraphy or cardiac catheter examination is contemplated.

- Image tests such as chest and abdominal X-ray photographs, ultrasonography (kidney echography), and abdominal CT is performed to examine renal deformities and complications. Atrophic kidney indicates long-term kidney damage, but not acute lesion, making it hard to expect recovery of kidney function. Moreover, renal carcinoma complicates atrophic kidney more often than usually.

- Physicians do not omit psychiatric care. 